Revista Brasileira de Agricultura Irrigada v.12, no.2, p. 2510 - 2518, 2018

ISSN 1982-7679 (On-line)

Fortaleza, CE, INOVAGRI - http://www.inovagri.org.br

DOI: $10.7127 /$ rbai.v12n200768

Protocolo 768.18 - 21/09/2017 Aprovado em 13/11/2017

\title{
ESPACIALIZAÇÃO DA EVAPOTRANSPIRAÇÃO DE REFERÊNCIA POR MEIO DA EQUAÇÃO HARGREAVES E SAMANI AJUSTADA PARA O ESTADO DO CEARÁ
}

\author{
Juarez Cassiano de Lima Junior ${ }^{1}$, Francisco Dirceu Duarte Arraes ${ }^{2}$, Kleber Gomes Macêdo ${ }^{3}$, \\ Joaquim Branco de Oliveira ${ }^{4}$, Willame Candido Oliveira ${ }^{3}$
}

\begin{abstract}
RESUMO
Objetivou-se espacializar a evapotranspiração de referência por meio de Sistema de Informação Geográfica utilizando a equação de Hargreaves e Samani ajustada para o estado do Ceará. Foram utilizados dados de vinte estações meteorológicas convencionais provenientes do Instituto Nacional de Meteorologia, localizadas em cidades dos estados: Ceará, Rio Grande do Norte, Pernambuco, Piauí e Paraíba. Foram utilizados dados mensais de insolação, velocidade do vento, umidade relativa e temperaturas máxima, média e mínima do ar. Para avaliação dos resultados da espacialização do modelo ajustado com o original foram utilizados os índices estatísticos: Raiz quadrada do erro médio, erro percentual médio e razão entre as médias. Nos resultados obtidos com a equação ajustada o parâmetro $\alpha$ oscilou entre 0,004 e 0,0023 , sendo o valor máximo seu valor original, e o expoente $\beta$, que possui valor original de 0,5 , apresentou variação de 0,49 a 1,0 . Os maiores valores de raiz quadrada do erro médio e erro percentual médio foram constatados no mês de maio, 1,11 e 22,61 respectivamente, bem como a maior subestimativa, alcançando $89,5 \%$ do valor estimado com a equação original para o mesmo mês.
\end{abstract}

Palavras-chave: demanda hídrica atmosférica, sistema de informação geográfica, semiárido

\section{SPATIZATION OF REFERENCE EVAPOTRANSPIRATION BY HARGREAVES AND SAMANI EQUATION ADJUSTED TO THE STATE OF CEARÁ}

\begin{abstract}
The objective of this study was to spatialize the reference evapotranspiration by means of Geographic Information System using an equation of Hargreaves and Samani adjusted for the state

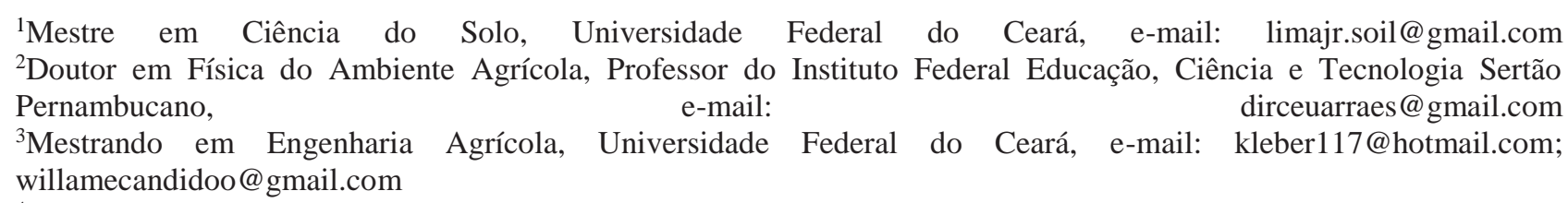
${ }^{4}$ Professor Dr. do Instituto Federal Educação, Ciência e Tecnologia do Ceará, e-mail: joaquimbrancodeoliveira@gmail.com
\end{abstract}




\section{Lima Junior et al.}

of Ceará. Meteorological data from the National Institute of Meteorology, located in cities of the states: Ceará, Rio Grande do Norte, Pernambuco, Piauí and Paraíba. Data is sunshine, wind speed, relative humidity average and maximum, average and minimum temperatures. To evaluate the results of the spatialization of the adjusted version with the original, the statistical indices were used: Square root of mean error, mean percentage and ratio of means. Our results obtained with an equation adjusted the parameter to oscillate between 0.004 and 0.0023 , being the maximum value of its original value, and the exponent $\beta$, which has original value of 0.5 , presented variation from 0.49 to 1.0. The highest values of square root mean error and mean error were observed in May, 1.11 and 22.61 respectively, as well as the highest underestimate, reaching $89.5 \%$ of the estimated value with an original equation for the same month.

Keywords: atmospheric water demand, geographic information system, semiarid

\section{INTRODUÇÃO}

Evaporação e transpiração são fenômenos que ocorrem simultaneamente e não possuem uma forma simplificada de distinção, em função disso o processo de transferência de água para a atmosfera é denominado de evapotranspiração (ET). Diante disso, o acompanhamento de forma precisa desse fenômeno é de fundamental importância no manejo de sistemas de irrigação e recursos hídricos, visto que, a evapotranspiração tem alta variação espacial e temporal, corroborando com tal afirmação Silva et al. (2013) verificou que por meio da espacialização e geração de mapas foi possível verificar o comportamento da ET nas mais remotas áreas estudadas no estado do Ceará.

Muitos modelos propostos para a estimativa da evapotranspiração de referência $\left(\mathrm{ET}_{\mathrm{o}}\right)$ não podem ser aplicados em climas diferentes daqueles em que foram originalmente desenvolvidos. A fim de resolver esse problema a Organização das Nações Unidas para Alimentação e Agricultura (FAO). Propôs o modelo de Penman-Monteith (PM) como padrão na estimativa da evapotranspiração de referência (ALLEN et al., 1998).

Allen et al. (1998) recomendam o uso da equação de Hargreaves e Samani (HARGREAVES; SAMANI, 1985) como alternativa para a estimativa da $\mathrm{ET}_{\mathrm{o}}$ quando há falta de elementos climáticos requeridos pelo modelo padrão (Penman-Monteith FAO 56). Quando ajustado, o modelo Hargreaves e Samani (HS) fornece dados confiáveis da evapotranspiração de referência, quando comparado com o modelo padrão, para o intervalo de cinco ou mais dias (TRAJKOVIC, 2007; THEPADIA; MARTINEZ, 2012; JERSZURKI; SOUZA, 2013; LIMA JUNIOR et al., 2016; ARRAES et al., 2016).

Em um sistema hidrológico também se faz necessário a espacialização das variáveis que o compõem, dentre elas a $\mathrm{ET}_{\mathrm{o}}$ (XU et al., 2006). Vários autores vêm utilizando o Sistema de Informações Geográficas (SIG) para espacializar e facilitar a forma de como se observa a dinâmica entre elementos meteorológicos (BARDIN; PEDRO JÚNIOR; MORAES, 2010; CARGNELUTTI FILHO; MALUF; MATZENAUER, 2008; ROCHA et al., 2011). Metodologias de se estimar a $\mathrm{ET}_{\mathrm{o}}$ pelo modelo de Hargreaves e Samani utilizando Modelos Digitais de Elevação (MDE) foram desenvolvidos por Oliveira et al. (2013).

Diante do exposto o objetivo do presente trabalho foi espacializar a estimativa de ET $_{\mathrm{o}}$ utilizando da equação de Hargreaves e Samani (1985) ajustada para o estado do Ceará. 


\section{MATERIAL E METODOS}

O estudo foi realizado utilizando dados meteorológicos do estado do Ceará, localizado entre os paralelos $2,5^{\circ}$ e $8^{\circ}$ de latitude Sul e os meridianos $37^{\circ}$ e $42^{\circ}$ de longitude Oeste, na região Nordeste do Brasil, com área total de $146.348,6 \mathrm{~km}^{2}$. De acordo com a classificação climática de Koppen, a região apresenta três tipos de clima: BSw'h', Aw' e Cw', sendo que em $80 \%$ da área existe predominância do clima BSw'h' (semiárido quente) (OLIVEIRA et al., 2013).

Foram utilizados dados provenientes de estações meteorológicas convencionais de vinte municípios (Figura 1 e Tabela 1). Dados do Instituto Nacional de Meteorologia (INMET) são: velocidade do vento $(\mathrm{u})$, insolação $(\mathrm{n})$, temperatura máxima $\left(\mathrm{T}_{\max }\right)$, temperatura mínima $\left(\mathrm{T}_{\min }\right)$, temperatura média $\left(\mathrm{T}_{\mathrm{med}}\right)$, e umidade relativa (UR).

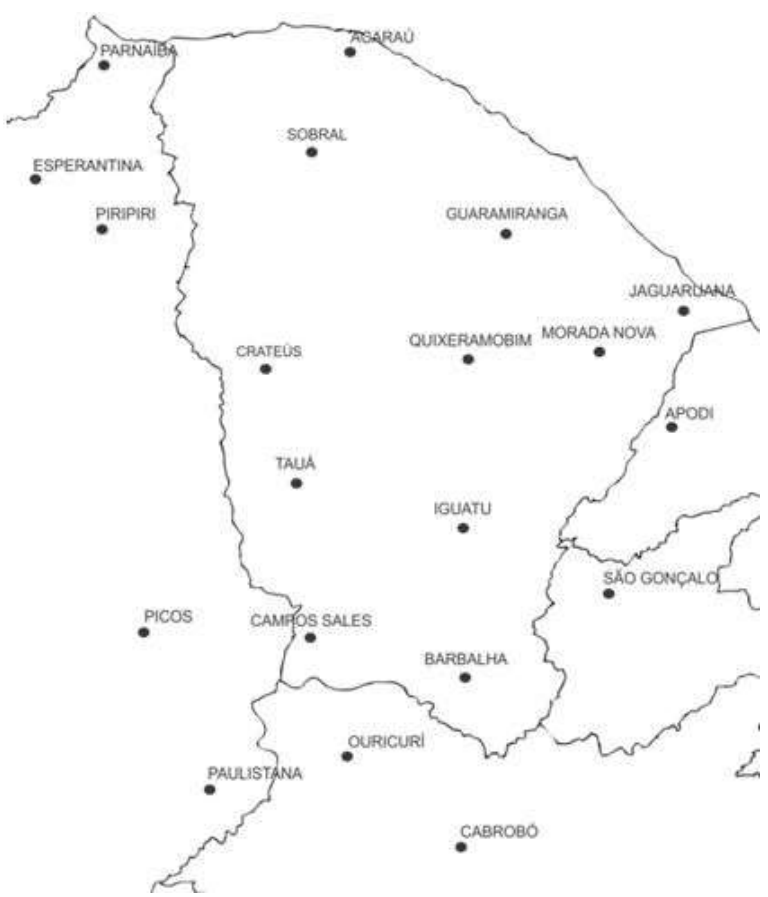

Figura 1. Localização das estações meteorológicas (LIMA JUNIOR et al., 2016).

Tabela 1 - Localização das estações meteorológicas utilizadas no estudo

\begin{tabular}{clcccc}
\hline Estações & UF & Latitude $\left(^{\circ}\right)$ & Longitude $\left(^{\circ}\right)$ & Altitude $(\mathrm{m})$ & Período \\
& & & & & \\
\hline Acaraú & CE & $-2,88$ & $-40,14$ & 16,5 & $1976 / 2011$ \\
Barbalha & CE & $-7,31$ & $-39,3$ & 409 & $1973 / 2014$ \\
Campos Sales & CE & $-7,00$ & $-40,38$ & 583,50 & $1962 / 2014$ \\
Crateús & CE & $-5,16$ & $-40,66$ & 296,82 & $1961 / 2014$ \\
Guaramiranga & CE & $-4,28$ & $-39,00$ & 870,67 & $1961 / 2014$ \\
Iguatu & CE & $-6,36$ & $-39,29$ & 217,60 & $1961 / 2014$ \\
Jaguaruana & CE & $-4,87$ & $-37,76$ & 11,71 & $1969 / 2014$ \\
Morada Nova & CE & $-5,11$ & $-38,36$ & 43,62 & $1962 / 2014$ \\
Quixeramobim & CE & $-5,16$ & $-39,38$ & 79,50 & $1961 / 2014$ \\
Sobral & CE & $-3,73$ & $-40,33$ & 109,62 & $1961 / 2014$ \\
Tauá & CE & $-6,00$ & $-40,41$ & 398,77 & $1964 / 2014$
\end{tabular}




\begin{tabular}{cccccc} 
Apodi & RN & $-5,60$ & $-37,80$ & 150,00 & $1962 / 2014$ \\
Cabrobó & PE & $-8,51$ & $-39,33$ & 341,46 & $1963 / 2014$ \\
Ouricuri & PE & $-7,90$ & $-40,04$ & 459,28 & $1975 / 2014$ \\
Esperantina & PI & $-3,90$ & $-42,25$ & 87,05 & $1979 / 2014$ \\
Parnaíba & PI & $-3,80$ & $-41,76$ & 79,50 & $1971 / 2014$ \\
Paulistana & PI & $-8,13$ & $-41,13$ & 374,22 & $1975 / 2014$ \\
Picos & PI & $-7,03$ & $-41,48$ & 207,93 & $1974 / 2014$ \\
Piripiri & PI & $-4,26$ & 41,78 & 161,12 & $1976 / 2014$ \\
São Gonçalo & PB & $-6,75$ & $-38,21$ & 233,06 & $1961 / 2014$ \\
\hline
\end{tabular}

Lima Junior et al. (2016)

Hargreaves e Samani (1985) propuzeram uma equação para estimativa da $\mathrm{ET}_{\mathrm{o}}$, em que os dados de entrada necessários são os valores de Temperatura máxima, minima e média do ar e da Radiação no topo da atmosfera (equação 1):

$$
\text { ETo }=\alpha \times(\text { T max } T \text { min })^{\beta} \times(\text { Tmed }+17,8) \times R a \times 0,408
$$

Em que: $\alpha$ - parâmetro empírico; $\beta$ parâmetro empírico exponencial; $\mathrm{T}_{\max }-$ Temperatura máxima do ar; $\mathrm{T}_{\min }-$ Temperatura mínima do ar; $\mathrm{T}_{\text {med }}-$ Temperatura média do ar $\left({ }^{\circ} \mathrm{C}\right) ; \mathrm{R}_{\mathrm{a}}-$ Radiação no topo da atmosfera, (MJ m$\left.{ }^{-2} \mathrm{dia}^{-1}\right)$.

A radiação solar no topo da atmosfera foi determinada utilizando a metodologia proposta por Allen et al. (1998), que considera o dia 15 de cada mês como referência no cálculo da média da $\mathrm{ET}_{\mathrm{o}}$ para cada mês.
Foi feita a espacialização dos coeficientes ajustados e das estimativas de ETo, sendo que as estimativas foram comparadas com a equação de Hargreaves e Samani original (1985). Foram utilizadas as metodologias desenvolvidas por Oliveira et al. (2013), utilizando um modelo de regressão linear múltipla, tendo altitude, longitude e latitude, como variáveis independentes, e temperatura medida como variável dependente, baseadas no seguinte modelo quadrático:

$$
\text { Tmed }=A_{0}+A_{1} \cdot h+A_{2} \cdot h^{2}+A_{3} \cdot \lambda+A_{4} \cdot \lambda^{2}+A_{5} \cdot \varphi+A_{6} \cdot \varphi^{2}+A_{7} j \cdot \varphi+A_{8} \cdot h \lambda+A_{9} \cdot h \lambda
$$

Em que:

Tmed - Temperatura média mensal $(\mathrm{i}=1,2$, 3..., 12);

$\lambda$ - longitude em graus;

$\varphi$ - latitude em graus;

$\mathrm{h}$ - altitude em metros;

An - coeficientes da regressão.

Os coeficientes de entrada da equação de Hargreaves e Samani (1985), ajustados para o estado do Ceará, estão dispostos na tabela 2, sendo os mesmos obtidos por Lima Junior et al. (2016), foram aplicados na espacialização da $\mathrm{ET}_{\mathrm{o}}$ na forma de mapas em escala mensal, utilizando o software Idrisi Andes $\odot$, por meio das mesmas metodologias empregadas por Oliveira et al. (2013).

Tabela 2 - Valores dos coeficientes empíricos da equação de Hargreaves e Samani (HS), calibrados para o estado do Ceará.

\begin{tabular}{ccc}
\hline Cidades & $\alpha$ & $\beta$ \\
\hline Acaraú & 0,0007 & 1,03 \\
Barbalha & 0,0013 & 0,68
\end{tabular}




$\begin{array}{ccc}\text { Campos Sales } & 0,0004 & 1,19 \\ \text { Crateús } & 0,0004 & 1,15 \\ \text { Guaramiranga } & 0,0019 & 0,51 \\ \text { Iguatu } & 0,0008 & 0,95 \\ \text { Jaguaruana } & 0,0009 & 0,91 \\ \text { Morada Nova } & 0,0012 & 0,73 \\ \text { Quixeramobim } & 0,0008 & 0,97 \\ \text { Sobral } & 0,0011 & 0,74 \\ \text { Tauá } & 0,0008 & 0,92\end{array}$

Lima Junior et al. (2016)

Todo o processamento do Modelo Digital de Elevação (MDE) contou com auxilio de dados de controle topográficos, tornando possível a validação dos dados gerados. Os dados de radar foram coletados durante uma missão de 11 dias, posteriormente processados com a metodologia descrita por Rabus et al. (2003). Os Dados da Missão Topográfica pelo Radar Shuttle (acrónimo em inglês SRTM) são referenciados ao elipsoide WGS84. Sendo os mesmos adquiridos através da internet. A análise dos resultados foi realizada de acordo com os índices estatísticos, raiz quadrada do erro médio (RMSE), erro percentual médio (EPE), e razão entre as médias em porcentagem $(\xi)$ equações de 3 a 5 respectivamente.

$$
R M S E=\sqrt{\frac{\sum_{i=1}^{N}\left(\begin{array}{ll}
Y i & X i
\end{array}\right)^{2}}{n}}
$$

$$
\begin{gathered}
E=\frac{\sum_{i=1}^{N}(Y i \quad X i)}{Y i} \times 100 \\
\xi=\frac{\overline{Y i}}{\overline{X i}}
\end{gathered}
$$

Em que:

Yi- valores médios obtidos pelos modelos propostos;

$\mathrm{Xi}$ - valores médios obtidos pelo modelo padrão.

\section{RESULTADOS E DISCUSÃO}

A espacialização dos coeficientes ajustados se fez permitir um melhor entendimento do comportamento e da sensibilidade dos valores parametrizados. No ajuste dos parâmetros é perceptível que eles possuem comportamento inversamente proporcional, pois o parâmetro alfa apresenta oscilação entre 0,0004 e 0,0023 (Figura 2). 

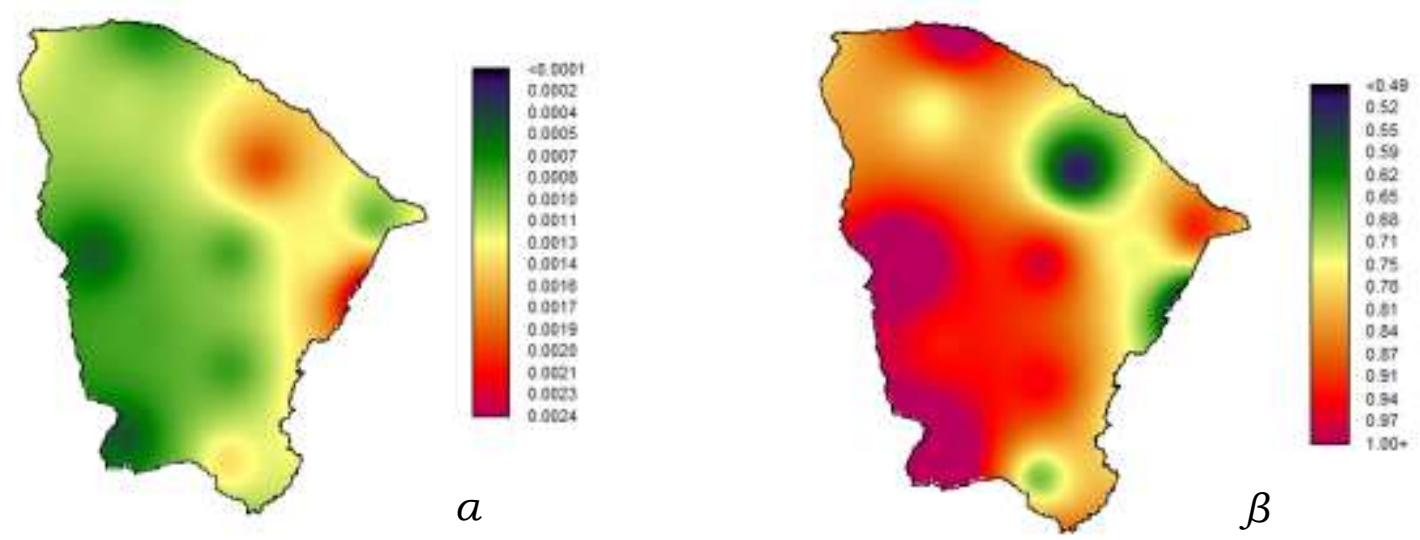

Figura 2. Distribuição espacial dos coeficientes alfa e beta ajustados.

Próximo à estação de Guaramiranga o coeficiente alfa mostra seu maior valor, tal resultado pode ser atribuído às baixas temperaturas do ar registradas na região, em função da alta altitude. Mesmo comportamento que ocorre no extremo leste do estado, nas proximidades da chapada do Apodi, sendo que, a possível causa dessa representação não é a altitude, mas sim a falta de uma estação localizada na parte mais alta da região. A estação meteorológica convencional de Apodi - RN está localizada a apenas $150 \mathrm{~m}$ de altitude, o que não representa de forma concreta a influência da formação geográfica da chapada no clima da região, segundo Mendonça e Danni-Oliveira (2007) o ar tem sua densidade alterada com a altitude, podendo em função de sua compressão apresentar isotermia (diminuição da amplitude térmica), ou ate mesmo inversão de temperatura.

Como a temperatura é principal dado de entrada do modelo HS é possível assumir a hipótese que no restante do estado o coeficiente $\alpha$ vai diminuindo conforme se afasta da região serrana e/ou em função das velocidades de vento e temperaturas do ar mais elevadas registradas no restante do estado.

O parâmetro $\beta$ oscilou de 0,49 a 1,0 aproximadamente, apresentou em praticamente todo o estado seus valores mais elevados. Por ser o expoente da diferença de temperatura modelo de HS, representando assim a amplitude térmica, o expoente beta evidência uma maior sensibilidade, expressando assim sua maior importância entre os parametros ajustados, visto que a região de estudo possui clima bem definido, classificada em sua maior parte como semiárida, com altas temperaturas durante quase todo o ano.

É importante enfatizar que na variação do ajuste dos parâmetros da equação HS, os valores originais estiveram nos limites, sendo o coeficiente alfa original o valor máximo obtido no ajuste $(0,0023)$ e o expoente beta original $(0,5)$ muito próximo do menor valor ajustado. Lima Junior et al. (2016) verificou que quando ajustado somente coeficiente alfa da equação HS, a qualidade das estimativas foi prejudicada, valorizando assim o ajuste local do expoente $\beta$, melhorando a adaptação da equação empirica para região de estudo.

A espacialização da evapotranspiração de referência permitiu a compreensão da variablididade espacial e temporal desse fenômeno no estado do Ceará em toda sua extensão. Xu et al. (2006), explanam que mapas de distribuição espacial guarnecem informações muitos valiosas na gestão e planejamento de recursos hídricos em uma bacia ou região, em função da grande importância que a $\mathrm{ET}_{\mathrm{o}}$ possui como elemento do ciclo hidrológico. 
Analisando a variação espacial e temporal das estimativas de $\mathrm{ET}_{\mathrm{o}}$, se faz possivel a compreensão do comportamento dos dois modelos em todo estado do Ceará. O modelo original e o ajustado apresentaram suas maiores demandas evapotranspirativas nos meses de Setembro e Outubro, respectivamente (Figura 3), aproximadamente $8,0 \mathrm{~mm}$ para $\mathrm{HS}$ original e $10,0 \mathrm{~mm}$ para $\mathrm{HS}$ ajustado no extremo oeste da região estudada. Tabari et al. (2013) espacializando a estimativa da evapotranspiração de referência no sudeste do Irã (região mais árida do país) utilizando a equação de Blaney-Criddle ajustada encontrou as maiores estimativas de $\mathrm{ET}_{\mathrm{o}}(11,0 \mathrm{~mm})$ para os meses de junho, julho $\mathrm{e}$ agosto.
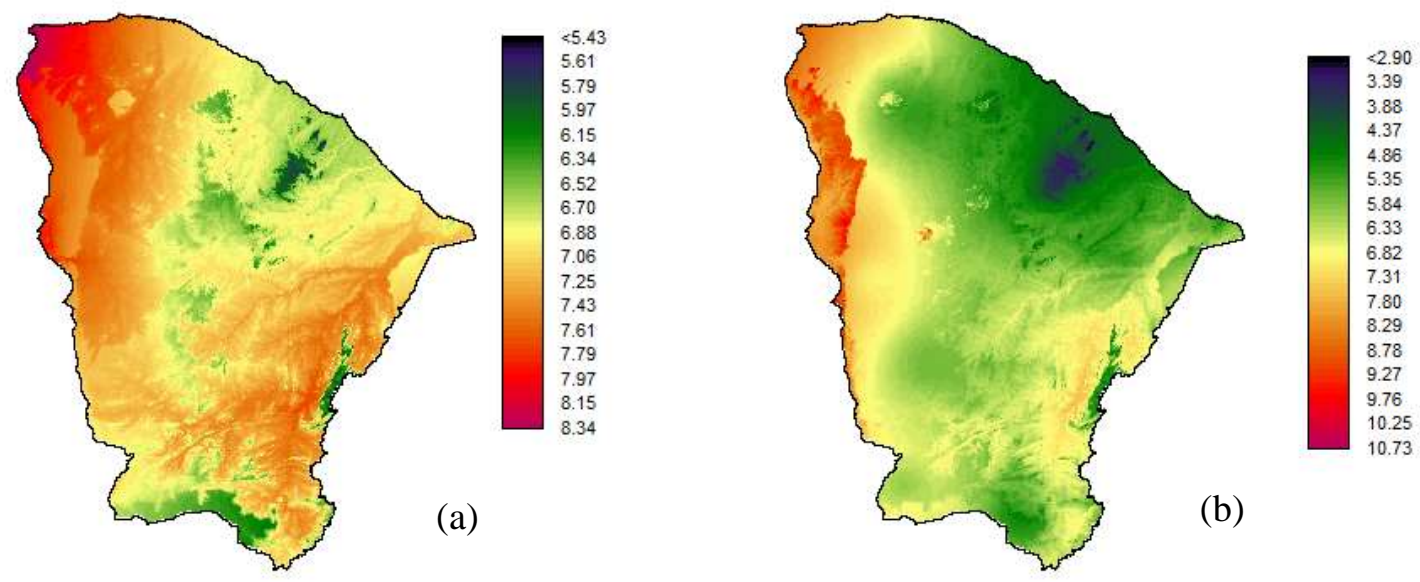

(b)

Figura 3. Distribuição espacial da $\operatorname{ET}_{\mathrm{o}}\left(\mathrm{mm} \mathrm{dia}^{-1}\right)$ no mês de máxima demanda para Hargreaves Original (a); Hargreaves ajustado (b).

Nos meses de pico de $\mathrm{ET}_{\mathrm{o}}$ o local que apresentou menor demanda evapotranspirativa para a modelagem com a equação HS original e ajustado foi a região da serra de Guaramiranga, o que mais uma vez pode ser justificado pela sua altitude elevada e suas baixas normais de temperatura do ar, nos meses de Março e Maio a menor demanda de $\mathrm{ET}_{\mathrm{o}}$ pode ser observada no litoral do estado.

Para os meses de maior demanda evapotranspirativa foram estimados pela espacialização valores superiores aos comumente registrados nas estações (próximos de 8,0 mm). Esse comportamento na estimativa da espacialização da $\mathrm{ET}_{\mathrm{o}}$ com a equação ajustada pode ser justificado por uma diminuição de representatividade das estações localizadas nos extremos leste e oeste do estado avaliado, pois nos limites entre Ceará e Piaui existe uma grande elevação geográfica com mais de 800 m (Chapada da Ibiapaba), a qual nenhuma estação meteorologica com historico suficiente nessa altitude elevada, em função disso a espacialização naquela região pode ter sido prejudicada pela falta de precisão da interpolação das estimativas do noroeste do Ceará com as do Leste do Piauí, inclusive comportamento semelhante foi verificado por Oliveira et al. (2013).

Analisando a variação da razão entre as médias (Tabela 3), que oscilou entre 89,5 e $110,6 \%$ para os meses de Maio e Novembro respectivamente percebe-se que no mês de Maio a equação original subestimou (10,5\%) os resultados obtidos com o modelo calibrado, tendo em vista que somente nos meses de Agosto e Setembro as equações comparadas praticamente não diferiram entre si, meses em que ocorrem rotineiramente as maiores temperaturas no estado do Ceará, e como o modelo de HS é baseado em temperatura, pouco sofreu alteração no ajuste feito. Ainda 


\section{Lima Junior et al.}

na mesma tabela é observado que o RMSE variou de 0,54 nos meses de fevereiro e abril, ate 1,11 no mês de maio. O EPM oscilou entre 9,17 (fevereiro) e 22,1 (maio), resultados que dão ênfase a uma maior diferença entre as equações utilizadas no mês de maio.

Tabela 3. Razão entre as médias ( $\xi$ ), raiz quadrada do erro médio (RMSE) e erro percentual médio (EPM) entre a $\mathrm{ET}_{\mathrm{o}}$ estimada pelo modelos digitais de elevação utilizando as equações de HS original (1985) e HS calibrados o alfa e beta.

\begin{tabular}{cccc}
\hline Mês & $\begin{array}{c}\text { RMSE } \\
\left(\mathrm{mm} \mathrm{dia}^{-1}\right)\end{array}$ & $\begin{array}{c}\text { EPM } \\
(\%)\end{array}$ & $\xi(\%)$ \\
\hline Janeiro & 0,75 & 11,9 & 105,8 \\
Fevereiro & 0,54 & 9,17 & 102 \\
Março & 0,64 & 12,72 & 113 \\
Abril & 0,54 & 10,35 & 107,4 \\
Maio & 1,11 & 22,61 & 89,5 \\
Junho & 0,47 & 10,55 & 103,5 \\
Julho & 0,62 & 12,58 & 102,5 \\
Agosto & 0,57 & 9,39 & 99,9 \\
Setembro & 0,74 & 10,45 & 99,4 \\
Outubro & 0,70 & 11,19 & 97,3 \\
Novembro & 0,86 & 12,26 & 110,6 \\
Dezembro & 0,70 & 11,67 & 105,2 \\
\hline
\end{tabular}

\section{CONCLUSÃO}

A espacialização da demanda evapotranspirativa no estado do Ceará apresenta resultados que possibilitam uma melhor compreensão espacial e temporal desse fenômeno, mostrando que os resultados diferentes obtidos com a calibração evidenciam que a metodologia original não apresenta resultados satisfatórios para todas as regiões e épocas do ano no Ceará.

A pouca quantidade de estações meteorológicas na região de estudo diminuiu a qualidade das estimativas espacializadas, ao mesmo tempo em que justificou a espacialização da evapotranspiração de referência, a fim de se entender melhor tal fenômeno em regiões com características geográficas e/ou climáticas discrepantes.

\section{REFERÊNCIAS}

ALLEN, R. G; PEREIRA, L. S; RAES, D; SMITH, M. Crop evapotranspiration: guidelines for computing water requeriments: FAO, 1998. 300 p. (FAO, Irrigation and Drainage Paper, 56)

ARRAES, F. D. D; LIMA JUNIOR, J. C; DE OLIVEIRA, J. B; DE MACÊDO, K. G; DE SOUSA COURAS, Y; e DE OLIVEIRA, W. C. Parametrização da equação de hargreavessamani para o estado do pernambuco-brasil. Revista brasileira de agricultura irrigada, v. 10 , n. 1, p. 410, 2016.

BARDIN, L.; PEDRO JÚNIOR, M. J. ; MORAIS, J. F. L. Estimativa das Temperaturas máximas e mínimas do ar para a região do Circuito das Frutas, SP. Revista Brasileira de Engenharia Agrícola e Ambiental, v. 14, n. 6, p. 618-624, 2010. 
CARGNELUTTI FILHO, A; MALUF, J. R. $\mathrm{T}$; MATZENAUER, R. Coordenadas geográficas na estimativa das temperaturas máximas e médias decendiais do ar no Estado do Rio Grande do Sul. Ciência Rural, v. 38, n. 9, p. 2448-2456, 2008.

HARGREAVES, G. H; SAMANI, Z. A. Reference crop evapotranspiration from temperature. Applied Engineering in Agriculture, v. 01, n. 02, p. 96-99, 1985.

JERSZURKI, D; SOUZA, J. L. M. Parametrização das equações de Hargreaves \& Samani e Angströn-Prèscott para estimativa da radiação solar na região de Telêmaco Borba, Estado do Paraná. Ciência Rural, v. 43, n. 3, p. 383-389, 2013.

LIMA JUNIOR, J. C; ARRAES, F. D; OLIVEIRA, J. B; NASCIMENTO, F. A. L; DE MACÊDO, K. G.. Parametrização da equação de Hargreaves e Samani para estimativa da evapotranspiração de referência no Estado do Ceará, Brasil. Revista Ciência Agronômica, v. 47, n. 3, p. 447, 2016.

MENDONÇA, F; DANNI-OLIVEIRA, I, M. Climatologia: noções básicas e climas do Brasil. v. 1. São Paulo: Oficina de Texto, 2007. 206 p.

OLIVEIRA， J. B.; ARRAES， F. D. D.; VIANA, P. C. Methodology for the spatialisation of a reference evapotranspiration from SRTM data. Revista Ciência Agronômica, Fortaleza, v. 44, n. 3, p. 445-454, jul-set, 2013.

RABUS, B; EINEDER, M; ROTH, A; BAMLER, R. The Shuttle Radar Topography Mission - a new class of digital elevation models acquired by spaceborne radar. ISPRS. Journal of Photogrammetry \& Remote Sensing, v. 57, n. 4, p. 241-262, 2003.
ROCHA, É. J. T; EVANGELISTA, S. R. M; FUCK JUNIOR, S. C. F; GONDIM, R S. Estimativa da $\mathrm{Et}_{\mathrm{o}}$ pelo modelo PenmanMonteith FAO com dados mínimos integrada a um Sistema de Informação Geográfica. Revista Ciência Agronômica, v. 42, n. 1, p. 75-83, 2011.

SILVA, M. G.; ARRAES, F. D. D.; LEDO, E. R. F.; SANTOS, N. T.; AILTON, J. Avaliação da evapotranspiração de referência por PenmanMonteith usando dados climáticos mínimos no sertão do Ceará, Agro@mbiente, v: 7,n:3; p: 284-293, 2013.

TABARI, H; TALAEE, P. H; SOME, B. S. Spatial modelling of reference evapotranspiration using adjusted BlaneyCriddle equation in an arid environment, Hydrological Sciences Journal, DOI:10.1080/02626667.2012.755265. 2013.

THEPADIA, M; MARTINEZ, C. J. Regional Calibration of Solar Radiation and Reference Evapotranspiration Estimates with Minimal Data in Florida. Journal of Irrigation and Drainage Engineering, v. 138, n. 2, p. 111119, 2012.

TRAJKOVIC, S. Hargreaves versus PenmanMonteith under humid conditions. Journal of Irrigation and Drainage Engineering, v. 133, n. 1, p. 38-42, 2007.

VALIPOUR, M. Temperature analysis of reference evapotranspiration models. Meteorological

XU, C.Y; GONG, L; JIANG, T; CHEN, D; SINGH, V. P. Analysis of spatial distribution and temporal trend of reference evapotranspiration and pan evaporation in Changjiang (Yangtze River) catchment. Journal of Hydrology, v. 327, n. 1/2, p. 8193, 2006. 